\title{
Aplicación de la técnica abdominal bracing en pacientes con lumbalgia inespecífica
}

\section{Abdominal brancing application for inespecific back pain}

\author{
DÍAZ, Ana C. ${ }^{1}$ \\ PEÑAFIEL, Andrea C. ${ }^{2}$ \\ LINARES, Juan I. ${ }^{3}$
}

\section{Resumen}

El estudio se realizó, en una clínica de la ciudad de Quito, con el objetivo de determinar la funcionalibilidad lumbar después de la aplicación de la técnica de Abdominal Brancing en pacientes con lumbalgia inespecífica. La investigación fue aplicativa, prospectiva y longitudinal. La muestra fue de 100 pacientes entre 20 a 30 años. Los criterios de inclusión fueron personas con lumbalgia inespecífica, que no tuviesen: escoliosis, artrosis rematoidea, miofibromialgia, dolor lumbar por causa traumatológica, hernias discales, dolores sacroiliacos. Al finalizar la implementación de Abdominal Bracing, si existió mejoría en la funcionalidad lumbar.

Palabras clave: abdominal bracing, lumbalgia inespecífica, control motor, músculo transverso abdominal.

\begin{abstract}
The study was carried out in a clinic in the city of Quito, with the aim of determining lumbar functionality after the application of the Abdominal Bracing technique in patients with non-specific low back pain. The research was applicative, prospective and longitudinal. The sample was 100 patients between 20 to 30 years old. The inclusion criteria were people with nonspecific low back pain, who did not have: scoliosis, rheumatoid osteoarthritis, myofibromyalgia, lumbar pain due to trauma, herniated discs, sacroiliac pain. At the end of the Abdominal Bracing implementation, if there was an improvement in lumbar functionality.

Key words: abdominal bracing, back pain, motor control, transverse abdominal muscle.
\end{abstract}

\section{Introducción}

Más del $80 \%$ de la población experimentan cierta lumbalgia en algún momento de su vida. La incidencia de este dolor es de 15 a 20\% (Maher et al., 2017). Las mujeres lo padecen más que los varones (Balagué et al., 2012).

La estabilización lumbar es primordial dentro del tratamiento kinesiológico de lumbago, debido a que la región lumbar es una de las zonas más móviles de la columna vertebral y por ende más inestable, por lo que en un lumbago se ve alterado el control muscular de los músculos estabilizadores (Panjabi, 2003).

\footnotetext{
${ }^{1}$ Magister en Terapia Manual ortopédica. Ciencias de la Rehabilitación, Salud y Gestión Deportiva. Universidad Andrés Bello. anidiaz1208@hotmail.com. ${ }^{2}$ Coordinadora de la carrera de fisioterapia de la UTA. Ciencias de la Salud. Universidad Técnica de Ambato. andyluna1986@live.com.

3 Policía Nacional del Ecuador. Departamento de docencia. Dirección Nacional de educación. Israelinares01@hotmail.com.
} 
La técnica de control motor Abdominal Bracing, evidencia mejoría de la estabilidad lumbopélvica, por un adecuado feedfordward del transverso abdominal en relación al movimiento de las extremidades inferiores (Lee et al., 2016).

Esta técnica consiste en la Co-contracción los músculos estabilizadores: transverso del abdomen y multífidos lumbares (Drysdale et al., 2004).

Hipótesis: Existe una mejoría en la funcionabilidad lumbar después de la aplicación de la técnica Abdominal Bracing.

\section{Metodología}

La investigación ejecutada fue de tipo aplicativa, prospectiva y longitudinal. La muestra obtenida fue de 100 pacientes entre 20 a 30 años, 36\% fueron hombres y $64 \%$ mujeres. Los criterios de inclusión fueron personas entre 20 a 30 años de edad con lumbalgia inespecífica, que no tuviesen: escoliosis, artrosis rematoidea, miofibromialgia, dolor lumbar por causa traumatológica, hernias discales, operaciones discales, espondilolistesis, dolores sacroiliacos.

Tabla 1

Alteración Postural de la columna Lumbar, según sexo

\begin{tabular}{|c|c|c|c|}
\hline & Femenino & Masculino & Totales \\
\hline Rectificación Lumbar & $19 \%$ & $21 \%$ & $40 \%$ \\
\hline Hiperlordosis Lumbar & $45 \%$ & $15 \%$ & $60 \%$ \\
\hline Total & $64 \%$ & $36 \%$ & $100 \%$ \\
\hline
\end{tabular}

Fuente: datos de los autores de la investigación

Al inicio de la investigación se realizó una evaluación postural estática para determinar alteraciones posturales de la columna vertebral, se observó $40 \%$ de rectificación lumbar y $60 \%$ de hiperlordosis lumbar en la muestra de estudio.

Previo a la aplicación de la técnica de Abdominal Bracing, a los pacientes se les administro el test de Oswestry, para cuantificar el porcentaje de limitación funcional de cada persona con lumbalgia inespecífica.

La técnica de control motor fue realizada dos veces por semana, durante 10 semanas, en un periodo de tiempo de 25 minutos de ejercicios.

En el estudio, se enseñó a cada paciente a co- contraer el transverso abdominal y los multífidos lumbares en las tres primeras semanas de tratamiento.

En la cuarta, quinta y sexta semana se añadió ejercicios de abducción horizontal de hombro (usando Theraband de color rojo), a la co-contracción de los músculos estabilizadores, previamente aprendidos en las anteriores semanas.

En la séptima, octava y novena semana se añadió ejercicios de flexión de cadera de $45^{\circ}$ (usando Theraband de color rojo).

En la última semana se mantenía el control motor en posiciones cotidianas, como sedestación, bipedestación y caminar. Y se realizó otra evaluación del porcentaje de funcionalidad lumbar, mediante el test de Oswestry.

El Análisis estadístico se lo realizó sobre la base de medidas de tendencia central, de dispersión, y prueba "T" de comparación de medias relacionadas, usando las herramientas informáticas de Microsoft Excel versión 2010 y SPSS versión 23. 


\section{Resultados}

Tabla 2

Media y medidas de dispersión del Test de Oswestry antes y después de la Aplicación de la técnica de Abdominal Bracing en pacientes con Lumbalgia

\begin{tabular}{|c|c|c|c|c|c|}
\hline & $\begin{array}{c}\text { Dato } \\
\text { Mínimo }\end{array}$ & $\begin{array}{c}\text { Dato } \\
\text { Máximo }\end{array}$ & Media & $\begin{array}{c}\text { Desviación } \\
\text { estándar }\end{array}$ & $\begin{array}{c}\text { Media de error } \\
\text { estándar }\end{array}$ \\
\hline TEST DE OWESTRY antes & 18 & 92 & 54.24 & 25.21 & 3.56 \\
\hline TEST DE OWESTRY después & 10 & 60 & 34.12 & 18.41 & 2.6 \\
\hline
\end{tabular}

Fuente: datos de los autores de la investigación

El dato máximo de porcentaje de la limitación de la funcionalidad previo a la aplicación de la técnica de Abdominal Bracing fue de 92, mientras que después de la aplicación fue de 60.

La media del resultado del test de Oswetry antes de la aplicación de la técnica de control motor fue de 54.24, al final del estudio la media fue de 34.12.

Tabla 3

Prueba T student del Test de Oswestry antes y después de la Aplicación de la técnica de Abdominal Bracing en pacientes con Lumbalgia

\begin{tabular}{|c|c|c|c|c|c|}
\hline & Diferer & ionadas & \multirow[b]{3}{*}{ Media } & \multirow[b]{3}{*}{ Desv. Est. } & \multirow[b]{3}{*}{$p$} \\
\hline & \multicolumn{2}{|c|}{$\begin{array}{c}\text { 95\% Intervalo de confianza para la } \\
\text { diferencia }\end{array}$} & & & \\
\hline & Inferior & Superior & & & \\
\hline $\begin{array}{l}\text { [ Diferencia entre distancia post } \\
\text { extracción y distancia pre } \\
\text { extracción. }\end{array}$ & $-16,10$ & $-24,14$ & $-20,12$ & 14,14 & 0,000 \\
\hline
\end{tabular}

Fuente: datos de los autores de la investigación

Después de la aplicación de la técnica de Abdominal Bracing la limitación funcional se redujo en un promedio de 20, 12 puntos. Existió una mayor reducción de 24, 14 y la mínima reducción fue de 16,10 después del entrenamiento de control motor del músculo transverso del abdomen y multífidos. Se observa que existió un cambio significativo en la muestra de estudio al ser $p<0,05$.

\subsection{Discusión}

El mantenimiento de la postura está dado por el sistema nervioso central y periférico, el tono muscular, la propiocepción, el sistema vestibular, el sistema visual, la alineación estructural. Si algunos de estos elementos se encuentran alterados la postura se modifica y pueden provocar algias (M. Kim et al., 2016).

En el estudio, existió más prevalencia de mujeres que tenían problemas lumbares que hombres, esto concuerda con estadísticas y estudios previos que citan la prevalencia de lumbago en féminas en todos los grupos de edad, esta diferencia entre hombres y mujeres aumenta aún más después de la edad de la menopausia femenina (Wang, 2017; Wáng et al., 2016).

Además de los músculos de la zona lumbar en las mujeres, existe prevalencia de incontinencia urinaria y dolor lumbar por la disminución del control motor. Los ejercicios de estabilización que se centran en el músculo del suelo pélvico mejoran la incontinencia urinaria de estrés y el dolor lumbar en mujeres con dolor lumbar inespecífico crónico (DOUGHERTY, 1998; Ghaderi et al., 2016). 
Los resultados apoyan la hipótesis de que la intervención con Abdominal Bracing es eficaz en el tratamiento de dolor lumbar. Los pacientes mostraron una mejoría significativa en la incapacidad funcional medida por el test de Oswestry.

La cocontracción de la muscular abdominal previa a movimientos de la extremidad inferior es primordial para un adecuado reclutamiento muscular en los movimientos articulares (O'Sullivan, 2005). Es importante que el movimiento lateral compensatorio de la pelvis no se produzca durante los movimientos de la cadera. En estudios de la técnica de Abdominal Bracing con medición de actividad electromiográfica del glúteo medio, cuadrado lumbar (QL), oblicuo externo del abdomen (EO) y oblicuo interno. abdominis (IO). Esta técnica de control motor aumenta la actividad muscular de QL, EO e IO, músculos importantes para el control motor de la zona lumbar y abdominal (D.-W. Kim \& Kim, 2018; Tayashiki et al., 2016).

\section{Conclusiones}

Esta investigación demuestra que la técnica de Abdominal Bracing al ser aplicada por 10 semanas, 2 veces a la semana, mejoró la funcionabilidad de los pacientes que presentan lumbalgia inespecífica.

La región lumbar es una zona inestable, y el control motor del transverso abdominal y de los multífidos lumbares permite disminución de los síntomas de la lumbalgia en pacientes de 20 a 30 años.

\subsection{Conflictos de intereses}

Los autores declaran que no existen conflictos de intereses.

\section{References}

Balagué, F., Mannion, A. F., Pellisé, F., \& Cedraschi, C. (2012). Non-specific low back pain. The Lancet, 379(9814), 482-491.

DOUGHERTY, M. (1998). Current status of research on pelvic muscle strengthening techniques*1. Journal of Wound, Ostomy, and Continence Nursing : Official Publication of the Wound, Ostomy and Continence Nurses Society, 25(2), 75-83. https://doi.org/10.1016/s1071-5754(98)90092-4

Drysdale, C. L., Earl, J. E., \& Hertel, J. (2004). Surface Electromyographic Activity of the Abdominal Muscles During Pelvic-Tilt and Abdominal-Hollowing Exercises. Journal of Athletic Training, 39(1), 32-36.

Ghaderi, F., Mohammadi, K., Amir Sasan, R., Niko Kheslat, S., \& Oskouei, A. E. (2016). Effects of Stabilization Exercises Focusing on Pelvic Floor Muscles on Low Back Pain and Urinary Incontinence in Women. Urology, 93, 50-54. https://doi.org/10.1016/j.urology.2016.03.034

Kim, D.-W., \& Kim, T.-H. (2018). Effects of abdominal hollowing and abdominal bracing during side-lying hip abduction on the lateral rotation and muscle activity of the pelvis. Journal of Exercise Rehabilitation, 14(2), 226-230.

Kim, M., Kim, Y., Oh, S., Suh, D., Eun, S.-D., \& Yoon, B. (2016). Abdominal hollowing and bracing strategies increase joint stability in the trunk region during sudden support surface translation but not in the lower extremities. Journal of Back and Musculoskeletal Rehabilitation, 29(2), 317-325.

https://doi.org/10.3233/BMR-150633

Lee, A. Y., Baek, S. O., Cho, Y. W., Lim, T. H., Jones, R., \& Ahn, S. H. (2016). Pelvic floor muscle contraction and abdominal hollowing during walking can selectively activate local trunk stabilizing muscles. Journal of Back and Musculoskeletal Rehabilitation, 29(4), 731-739. https://doi.org/10.3233/BMR-160678 
Maher, C., Underwood, M., \& Buchbinder, R. (2017). Non-specific low back pain. The Lancet, 389(10070), 736747.

O'Sullivan, P. (2005). Diagnosis and classification of chronic low back pain disorders: Maladaptive movement and motor control impairments as underlying mechanism. Manual Therapy, 10(4), 242-255. https://doi.org/10.1016/j.math.2005.07.001

Panjabi, M. M. (2003). Clinical spinal instability and low back pain. Journal of Electromyography and Kinesiology, 13(4), 371-379. https://doi.org/10.1016/S1050-6411(03)00044-0

Tayashiki, K., Takai, Y., Maeo, S., \& Kanehisa, H. (2016). Intra-abdominal Pressure and Trunk Muscular Activities during Abdominal Bracing and Hollowing. International Journal of Sports Medicine, 37(2), 134-143. https://doi.org/10.1055/s-0035-1559771

Wang, Y. X. J. (2017). Menopause as a potential cause for higher prevalence of low back pain in women than in age-matched men. Journal of Orthopaedic Translation, 8, 1-4.

Wáng, Y. X. J., Wáng, J.-Q., \& Káplár, Z. (2016). Increased low back pain prevalence in females than in males after menopause age: Evidences based on synthetic literature review. Quantitative Imaging in Medicine and Surgery, 6(2), 199-206.

\footnotetext{
Esta obra está bajo una Licencia Creative Commons Attribución-NoCommercial 4.0 International

\section{(cc) BY-NC}

